Supporting Information

\title{
Discovery and Preclinical Evaluation of BMS-711939, an Oxybenzylglycine Based PPAR $\alpha$ Selective Agonist
}

Yan Shi,* Jun Li, Lawrence J. Kennedy, Shiwei Tao, Andrés S. Hernández, Zhi Lai, Sean Chen, Henry Wong, Juliang Zhu, Ashok Trehan, Ngiap-Kie Lim, Huiping Zhang, Bang-Chi Chen, Kenneth T. Locke, Kevin M. O'Malley, Litao Zhang, Rai Ajit Srivastava, Bowman Miao, Daniel S. Meyers, Hossain Monshizadegan, Debra Search, Denise Grimm, Rongan Zhang, Thomas Harrity, Lori K. Kunselman, Michael Cap, Jodi Muckelbauer, Chiehying Chang, Stanley R. Krystek, Yi-Xin Li, Vinayak Hosagrahara, Lisa Zhang, Pathanjali Kadiyala, Carrie Xu, Michael A. Blanar, Robert Zahler, Ranjan Mukherjee, Peter T. W. Cheng, Joseph A. Tino

Research and Development, Bristol-Myers Squibb Company, 350 Carter Road, Hopewell, NJ 08540, U.S.A

* Tel: 1-609-466-5076. E-mail: yan.shi@bms.com

Contents

1. General methods

2. Experimental procedures and compounds characterization

3. In vivo assays

4. Pharmacokinetics protocols 
General Chemistry Methods. ${ }^{1} \mathrm{H}$ NMR spectra were recorded on a Bruker 400 or $500 \mathrm{MHz}$ machine with tetramethylsilane or residual protiated solvent used as a reference. ${ }^{13} \mathrm{C}$ NMR were recorded on a Bruker DRX-500 instrument operating at $125 \mathrm{MHz}$ with residual signals of the deuterated solvent used as a reference. LC-MS spectra were obtained on a Shimadzu HPLC and Micromass Platform using electrospray ionization. HRMS spectra were obtained on a Micromass LCT in lockspray with electrospray ionization. Analytical HPLC analyses were performed on a Shimadzu instrument using one of the following reverse phase methods, with UV detection set at $220 \mathrm{~nm}$ :

LC-MS: Phenomenex S5ODS 4.6mm x 50mmcolumn, gradient elution 0-100\% B/A over 4 min (solvent $\mathrm{A}=10 \% \mathrm{MeOH} / \mathrm{H}_{2} \mathrm{O}$ containing $0.1 \% \mathrm{H}_{3} \mathrm{PO}_{4}$, solvent $\mathrm{B}=90 \% \mathrm{MeOH} / \mathrm{H}_{2} \mathrm{O}$ containing $0.1 \% \mathrm{H}_{3} \mathrm{PO}_{4}$ ), flow rate $4 \mathrm{~mL} / \mathrm{min}$;

Analytical HPLC: Zorbax S5 SB-C18 $4.6 \mathrm{~mm} \times$ 75mm column, gradient elution 50-100\% B/A over 8min (solvent $\mathrm{A}=10 \% \mathrm{MeOH} / \mathrm{H}_{2} \mathrm{O}$ containing $0.2 \% \mathrm{H}_{3} \mathrm{PO}_{4}$, solvent $\mathrm{B}=90 \% \mathrm{MeOH} / \mathrm{H}_{2} \mathrm{O}$ containing $0.2 \% \mathrm{H}_{3} \mathrm{PO}_{4}$ ), flow rate $2.5 \mathrm{~mL} / \mathrm{min}$. The purity of all final compounds is determined by analytic HPLC method A and confirmed by analytic HPLC method B.

Preparative HPLC was carried out on an automated Shimadzu system using YMC ODS C18 5 $\mu \mathrm{m}$ preparative columns with mixtures of solvent $\mathrm{C}\left(10 \% \mathrm{MeOH} / 90 \% \mathrm{H}_{2} \mathrm{O} / 0.1 \%\right.$ TFA) and solvent D $\left(90 \% \mathrm{MeOH} / 10 \% \mathrm{H}_{2} \mathrm{O} / 0.1 \% \mathrm{TFA}\right)$ or of solvent $\mathrm{E}\left(10 \% \mathrm{CH}_{3} \mathrm{CN} / 90 \% \mathrm{H}_{2} \mathrm{O} / 0.1 \%\right.$ TFA) and solvent $\mathrm{F}\left(90 \% \mathrm{CH}_{3} \mathrm{CN} / 10 \% \mathrm{H}_{2} \mathrm{O} / 0.1 \%\right.$ TFA). All other reagents and solvents were obtained from commercial sources and were used without further purification.

2-Fluoro-5-hydroxybenzaldehyde (21a). To a $\quad-78 \quad{ }^{\circ} \mathrm{C}$ solution of 2-fluoro-5methoxybenzaldehyde $(16.2 \mathrm{~g}, 105.2 \mathrm{mmol})$ in dry $\mathrm{CH}_{2} \mathrm{Cl}_{2}(60 \mathrm{~mL})$ was added $\mathrm{BBr}_{3}(126.2 \mathrm{~mL}$ of a $1.0 \mathrm{M}$ solution in $\mathrm{CH}_{2} \mathrm{Cl}_{2}, 126.2 \mathrm{mmol}$ ) dropwise over $5 \mathrm{~min}$. The reaction was allowed to warm to $0{ }^{\circ} \mathrm{C}$ and stirred at $0{ }^{\circ} \mathrm{C}$ for $4 \mathrm{~h}$, (at this point HPLC/LCMS indicated that starting material had been completely consumed), then was cooled to $-78{ }^{\circ} \mathrm{C} . \mathrm{CH}_{2} \mathrm{Cl}_{2}(200 \mathrm{~mL})$ and water $(100 \mathrm{~mL})$ were added cautiously to quench the reaction, which was then warmed to RT. The organic layer was separated, and the aqueous layer was extracted with $\mathrm{CH}_{2} \mathrm{Cl}_{2}(3 \times 200 \mathrm{~mL})$. The combined organic extracts were dried $\left(\mathrm{MgSO}_{4}\right)$ and concentrated in vacuo to afford a brown residue, which was chromatographed $\left(120 \mathrm{~g} \mathrm{SiO}_{2}\right.$ ISCO column; continuous gradient from 1:99 to 40:60 EtOAc:hexanes) to give compound $2 \mathbf{a}(14.0 \mathrm{~g})$ as a brown solid. Spectra data for $2 \mathrm{a}:{ }^{1} \mathrm{H}$ $\operatorname{NMR}\left(\mathrm{CDCl}_{3}\right) \delta 10.22(\mathrm{~s}, 1 \mathrm{H}), 7.27-7.45(\mathrm{~m}, 2 \mathrm{H}), 7.11-7.15(\mathrm{~m}, 1 \mathrm{H}), 7.02(\mathrm{t}, 1 \mathrm{H}, \mathrm{J}=9.4 \mathrm{~Hz})$ ppm; ${ }^{19} \mathrm{~F} \mathrm{NMR}\left(\mathrm{CDCl}_{3}\right) \delta-131.95 \mathrm{ppm} ;{ }^{13} \mathrm{C} \mathrm{NMR}\left(\mathrm{CDCl}_{3}\right) \delta 188.66(\mathrm{~d}, \mathrm{~J}=5.1 \mathrm{~Hz}), 159.43(\mathrm{~d}, \mathrm{~J}$ $=251.8 \mathrm{~Hz}), 152.55,124.44(\mathrm{~d}, \mathrm{~J}=10.2 \mathrm{~Hz}), 123.90(\mathrm{~d}, \mathrm{~J}=7.6 \mathrm{~Hz}), 117.65(\mathrm{~d}, \mathrm{~J}=12.9 \mathrm{~Hz})$, 113.15 ppm; LRMS for $\mathrm{C}_{7} \mathrm{H}_{6} \mathrm{FO}_{2}:(\mathrm{M}+\mathrm{H})^{+}=141$.

Methyl (2-fluoro-5-hydroxybenzyl)glycinate (23a). To a stirred solution of glycine methyl ester hydrochloride $22(0.45 \mathrm{~g}, 3.6 \mathrm{mmol})$ in $\mathrm{MeOH}(5 \mathrm{~mL})$ was added $\mathrm{Et}_{3} \mathrm{~N}(0.50 \mathrm{~mL}, 3.6$ $\mathrm{mmol})$. Ater $5 \mathrm{~min}$, 2-Fluoro-5-hydroxybenzaldehyde (21a) $(0.25 \mathrm{~g}, 1.8 \mathrm{mmol})$ was added. After 
being stirred for $30 \mathrm{~min}$, the reaction mixture was cooled to $0{ }^{\circ} \mathrm{C}$, and $\mathrm{NaBH}_{4}(0.13 \mathrm{~g}, 3.6 \mathrm{mmol})$ was then added. The mixture was warmed to room temperature and was stirred for $1 \mathrm{~h}$. Volatiles were removed in vacuo at $45-50{ }^{\circ} \mathrm{C}$ from the reaction mass. The resulting residue was partitioned between EtOAc $(5 \mathrm{~mL})$ and water $(5 \mathrm{~mL})$, with the aqueous layer being washed again with EtOAc $(5 \mathrm{~mL})$. The combined organic extracts were washed with brine, dried $\left(\mathrm{Na}_{2} \mathrm{SO}_{4}\right)$, then concentrated in vacuo to afford the desired product as a pale-yellow solid $(0.36 \mathrm{~g}, 95 \%)$. LRMS for $\mathrm{C}_{10} \mathrm{H}_{13} \mathrm{FNO}_{3}:(\mathrm{M}+\mathrm{H})^{+}=214$.

Methyl $N$-(2-fluoro-5-hydroxybenzyl)- $N$-(methoxycarbonyl)glycinate (24a). To a stirred $0{ }^{\circ} \mathrm{C}$ solution of compound 23a $(0.25 \mathrm{~g}, 1.2 \mathrm{mmol})$ in THF $(1.3 \mathrm{~mL})$ and saturated aqueous $\mathrm{NaHCO}_{3}$ $(1 \mathrm{~mL})$ was added dropwise methyl chloroformate $(0.09 \mathrm{~mL}, 1.2 \mathrm{mmol})$ over 2 min under nitrogen. The mixturewas stirred at $0{ }^{\circ} \mathrm{C}$ for $10 \mathrm{~min}$ and extracted with EtOAc $(2 \times 2 \mathrm{~mL})$. The organic extracts were dried over anhydrous $\mathrm{Na}_{2} \mathrm{SO}_{4}$, filtered, and concentrated in vacuo to give crude product. The crude product was purified by flash column chromatography (silica gel 230 400 mesh, eluted with 0 to $100 \%$ EtOAc/hexanes) to give $0.32 \mathrm{~g}$ ( $98 \%$ yield) of $\mathbf{2 4 a}$ as a pale yellow oil. LRMS for $\mathrm{C}_{12} \mathrm{H}_{15} \mathrm{FNO}_{5}:(\mathrm{M}+\mathrm{H})^{+}=272$.

Methyl $\quad \mathrm{N}$-(5-((2-(4-chlorophenyl)-5-methyloxazol-4-yl)methoxy)-2-fluorobenzyl)- $N$ (methoxycarbonyl)glycinate (26a). To a solution of compound 24a $(0.32 \mathrm{~g}, 1.2 \mathrm{mmol})$ in $\mathrm{MeCN}(5 \mathrm{~mL})$ was added oxazole chloride $25 \mathrm{a}(0.29 \mathrm{~g}, 1.2 \mathrm{mmol})$ and anhydrous $\mathrm{K}_{2} \mathrm{CO}_{3}(0.50$ $\mathrm{g}, 3.6 \mathrm{mmol})$. The mixture was heated at $70{ }^{\circ} \mathrm{C}$ for $4 \mathrm{~h}$ under nitrogen and then cooled to room temperature. Sat. $\mathrm{NH} 4 \mathrm{Cl}(\mathrm{aq}) 5 \mathrm{~mL}$ was added. The mixture was extracted with EtOAC ( 2 x 10 $\mathrm{mL}$ ). The organic layer was dried over anhydrous $\mathrm{Na}_{2} \mathrm{SO}_{4}$, filtered, and concentrated in vacuo to yield the crude product. The crude product was purified by flash column chromatography ( $24 \mathrm{~g}$ silica gel, elution with $20-60 \% \mathrm{EtOAc} / \mathrm{heptane})$ to give $0.52 \mathrm{~g}$ (91\% yield) of product as a colorless oil. ${ }^{1} \mathrm{H}$ NMR $\left(\mathrm{CDCl}_{3}\right) \delta 7.91(\mathrm{~d}, 2 \mathrm{H}, \mathrm{J}=8.3 \mathrm{~Hz}), 7.38(\mathrm{~d}, 2 \mathrm{H}, \mathrm{J}=8.8 \mathrm{~Hz}), 6.98-7.0(\mathrm{~m}$, $1 \mathrm{H}), 6.94(\mathrm{dd}, 1 \mathrm{H}, \mathrm{J}=14.8,8.8 \mathrm{~Hz}), 6.85-6.90(\mathrm{~m}, 1 \mathrm{H}), 4.90(\mathrm{~s}, 2 \mathrm{H}), 4.55(\mathrm{~d}, 2 \mathrm{H}, \mathrm{J}=7.6 \mathrm{~Hz})$, $3.95(\mathrm{~d}, 2 \mathrm{H}, \mathrm{J}=15.7 \mathrm{~Hz}), 3.73(\mathrm{~s}, 2 \mathrm{H}$, one of carbamate's methoxy group rotamer), 3.69 (s, 4H, contains another carbamate's methoxy group rotamer and the ester methoxy group), 2.40 (s, 3H) ppm; ${ }^{19} \mathrm{~F}$ NMR $\left(\mathrm{CDCl}_{3}\right) \delta-128.31$ and $-128.63 \mathrm{ppm}$ (rotamers); ${ }^{13} \mathrm{C} \mathrm{NMR}\left(\mathrm{CDCl}_{3}\right) \delta 169.9$, 159.1, 156.9 and 156.7 (1C; rotamers), [156.6 and 154.7 (d, $\underline{\mathrm{C}}-\mathrm{F}, \mathrm{J}=241.6 \mathrm{~Hz}$ ), 156.4 and 154.5 $(\mathrm{d}, \underline{\mathrm{C}}-\mathrm{F}, \mathrm{J}=241.6 \mathrm{~Hz})]$ (rotamers), 154.6, 147.4, 136.2 and 136.1 (1C; rotamers), 131.9, 128.9 (2C), $127.3(2 \mathrm{C}), 125.8$ and 125.7 (1C; rotamers), 124.8-124.5 (1C; rotamers), 115.7-116.2 (1C; rotamers), 115.4 and 115.3 (1C; rotamers), 114.8 (1C; 2 peaks; rotamers), 62.6, 53.2 and 53.1 (1C; rotamers), 52.1, 48.2 and 47.9 (1C; rotamers), 45.4 and 45.2 (1C; rotamers), 10.4; HRMS for $\mathrm{C}_{23} \mathrm{H}_{23} \mathrm{ClFN}_{2} \mathrm{O}_{6}$, Calcd for $(\mathrm{M}+\mathrm{H})^{+}: 477.1229$, Found: 477.1225.

$N$-(5-((2-(4-chlorophenyl)-5-methyloxazol-4-yl)methoxy)-2-fluorobenzyl)- $N$ -

(methoxycarbonyl)glycine (3). The carbamate ester $26 \mathbf{a}(0.52 \mathrm{~g}, 1.1 \mathrm{mmol})$ was dissolved in 5 $\mathrm{ml}$ of THF. $\mathrm{NaOH}$ solution $(1 \mathrm{~N}, 5.5 \mathrm{ml}, 5.5 \mathrm{mmol})$ was added in. The reaction was stirred for 
$2 \mathrm{~h}$. Upon which time HPLC indicated completion of reaction. The reaction was neutralized with $1 \mathrm{~N} \mathrm{HCl}$ to $\mathrm{pH} \sim 2-3$, extracted with ethyl acetate $(3 \times 10 \mathrm{ml})$. The organic fractions were combined, dried over $\mathrm{MgSO}_{4}$, filtered, and concentrated to afford the crude. Preparative HPLC purification on a Shimadzu system using YMC ODS C18 $5 \mu \mathrm{m}$ preparative columns with mixtures of solvent $\mathrm{E} \quad\left(\begin{array}{llllll}10 \% & \mathrm{CH}_{3} \mathrm{CN} / 90 \% & \mathrm{H}_{2} \mathrm{O} / 0.1 \% & \mathrm{TFA}) & \text { and solvent } & \mathrm{F}\end{array}(90 \%\right.$ $\left.\mathrm{CH}_{3} \mathrm{CN} / 10 \% \mathrm{H}_{2} \mathrm{O} / 0.1 \% \mathrm{TFA}\right)$ to afford the title compound $3(0.47 \mathrm{~g}, 92 \%$ yield $)$ as a white solid. ${ }^{1} \mathrm{H}$ NMR (DMSO-d $\left.{ }_{6}\right) \delta 7.93(\mathrm{~d}, 2 \mathrm{H}, \mathrm{J}=8.4 \mathrm{~Hz}), 7.58(\mathrm{~d}, 2 \mathrm{H}, \mathrm{J}=8.4 \mathrm{~Hz}), 7.09-7.15(\mathrm{~m}, 1 \mathrm{H})$, 6.94-6.99 $(\mathrm{m}, 1 \mathrm{H}), 6.85-6.90(\mathrm{~m}, 1 \mathrm{H}), 4.97$ and $4.96(\mathrm{~s}, 2 \mathrm{H}$, rotamers), 4.47 and $4.46(\mathrm{~s}, 2 \mathrm{H}$, rotamers), $3.91(\mathrm{~s}, 2 \mathrm{H}), 3.60$ and $3.58\left(\mathrm{~s}, 3 \mathrm{H}\right.$, methoxy group rotamers), $2.43(\mathrm{~s}, 3 \mathrm{H}) \mathrm{ppm} ;{ }^{19} \mathrm{~F}$ NMR (DMSO-d ${ }_{6}$ ) $\delta-129.04$ and -129.15 ppm (rotamers); ${ }^{13} \mathrm{C}$ NMR (DMSO-d $\left.\mathrm{d}_{6}\right) \delta 170.8$ and 170.7 (1C; rotamers), 158.0, 156.2, [155.7 and 153.8; 1C; d, $\underline{\mathrm{C}}-\mathrm{F}, \mathrm{J}=238.0 \mathrm{~Hz}$ ), 155.6 and 153.7 $(1 \mathrm{C} ; \mathrm{d}, \underline{\mathrm{C}}-\mathrm{F}, \mathrm{J}=236.5 \mathrm{~Hz}$ )] (rotamers), 154.3 (1C; 2 peaks; rotamers), 147.9, 135.0, 132.0, 129.3 (2C), 127.4 (2C), 125.6, 125.0-125.5 (1C; rotamers), 115.8-116.1 (1C; rotamers), 115.4-115.8 (1C; rotamers), 114.56-114.93 (rotamers), 61.7, 52.7, 48.8 and 47.4 (1C; rotamers), 45.6 and 45.3 (1C; rotamers), 10.0; HRMS for $\mathrm{C}_{22} \mathrm{H}_{21} \mathrm{ClFN}_{2} \mathrm{O}_{6}$, Calcd for $(\mathrm{M}+\mathrm{H})^{+}: 463.1072$, Found: 463.1076; Anal. Calcd For $\mathrm{C}_{22} \mathrm{H}_{20} \mathrm{ClFN}_{2} \mathrm{O}_{6}$ : C, 57.08; H, 4.35; Cl, 7.66; F, 4.10; N, 6.05. Found: C, 57.09; H, 4.37; Cl, 7.62; F, 4.08; N, 6.19 .

\section{Alternative synthesis of 3.}

5-((2-(4-Chlorophenyl)-5-methyloxazol-4-yl)methoxy)-2- fluorobenzaldehyde (28). A solution of $21 \mathbf{a}(6.89 \mathrm{~g}, 28.6 \mathrm{mmol}), 25 \mathrm{a}(4.0 \mathrm{~g}, 28.6 \mathrm{mmol})$ and $\mathrm{K}_{2} \mathrm{CO}_{3}(11.84 \mathrm{~g}, 85.8 \mathrm{mmol})$ in MeCN $(50 \mathrm{~mL})$ was heated at $80{ }^{\circ} \mathrm{C}$ under $\mathrm{N}_{2}$ for $6 \mathrm{~h}$, at which point HPLC analysis indicated that the reaction was complete. The reaction was cooled to $\mathrm{RT}$. $\mathrm{CH}_{2} \mathrm{Cl}_{2}(50 \mathrm{~mL})$ was added and the mixture was stirred for $5 \mathrm{~min}$, then was filtered. The solid was washed with $\mathrm{CH}_{2} \mathrm{Cl}_{2}(20 \mathrm{~mL})$. The combined filtrates were concentrated in vacuo; the residue was chromatographed $\left(\mathrm{SiO}_{2} ; 120\right.$ g ISCO column; continuous gradient from 1-40\% EtOAc in hexanes) to give compound 28 compound $(9.76 \mathrm{~g}, 99 \%)$ as a white solid. ${ }^{1} \mathrm{H} \mathrm{NMR}\left(\mathrm{CDCl}_{3}\right) \delta 10.29(\mathrm{~s}, 1 \mathrm{H}), 7.89(\mathrm{~d}, 2 \mathrm{H}, \mathrm{J}=8.8$ $\mathrm{Hz}), 7.42(\mathrm{dd}, 1 \mathrm{H}, \mathrm{J}=5.3,3.6 \mathrm{~Hz}), 7.35(\mathrm{~d}, 2 \mathrm{H}, \mathrm{J}=8.3 \mathrm{~Hz}), 7.19-7.22(\mathrm{~m}, 1 \mathrm{H}), 7.06(\mathrm{t}, 1 \mathrm{H}, \mathrm{J}=$ $9.3 \mathrm{~Hz}), 4.94(\mathrm{~s}, 2 \mathrm{H}), 2.41(\mathrm{~s}, 3 \mathrm{H}) \mathrm{ppm} ;{ }^{19} \mathrm{~F} \mathrm{NMR}\left(\mathrm{CDCl}_{3}\right) \delta-131.71 \mathrm{ppm} ;{ }^{13} \mathrm{C} \mathrm{NMR}\left(\mathrm{CDCl}_{3}\right) \delta$ $186.7(\mathrm{~d}, \mathrm{~J}=7.6 \mathrm{~Hz}), 159.6(\mathrm{~d}, \mathrm{~J}=254.3 \mathrm{~Hz}), 159.1,154.7,147.5,136.1,131.5,128.9$ (2C), 127.3 (2C), 125.7, 124.5 (d, J = 10.2 Hz), 124.0 (d, J = 10.2 Hz), 117.5 (d, J = $22.9 \mathrm{~Hz}), 111.0$, $62.5,10.4$; LRMS for $\mathrm{C}_{18} \mathrm{H}_{14} \mathrm{ClFNO}_{3}:(\mathrm{M}+\mathrm{H})^{+}=346$.

\section{Methyl}

2-(5-((2-(4-chlorophenyl)-5-methyloxazol-4-yl)methoxy)-2fluorobenzylamino)acetate (29). To the above $\mathbf{2 5 a}(9.76 \mathrm{~g}, 28.3 \mathrm{mmol})$ in dry $\mathrm{MeOH}(50 \mathrm{~mL})$ was added glycine methyl ester hydrochloride $(4.31 \mathrm{~g}, 34.3 \mathrm{mmol})$ and $\mathrm{Et}_{3} \mathrm{~N}(5.17 \mathrm{ml}, 37.2$ $\mathrm{mmol}$ ), and the mixture was stirred under $\mathrm{N}_{2}$ at RT overnight. The reaction was then cooled to -5 ${ }^{\circ} \mathrm{C}$ and $\mathrm{NaBH}_{4}(1.41 \mathrm{~g}, 37.3 \mathrm{mmol})$ was added portionwise. The reaction was stirred at $0{ }^{\circ} \mathrm{C}$ for $30 \mathrm{~min}$, then was warmed to RT over $30 \mathrm{~min}$. $\mathrm{MeOH}(5 \mathrm{~mL})$ was then added dropwise at $0{ }^{\circ} \mathrm{C}$ to quench the reaction. The mixture was concentrated under vacuo. The residue was partitioned 
between EtOAc $(100 \mathrm{~mL})$ and water $(20 \mathrm{~mL})$. The organic phase was washed with brine $(20$ $\mathrm{mL})$, dried $\left(\mathrm{MgSO}_{4}\right)$, and concentrated in vacuo. The residue was chromatographed $\left(\mathrm{SiO}_{2} ; 120 \mathrm{~g}\right.$ ISCO column; continuous gradient from 0 to $100 \%$ EtOAc in hexanes) to afford compound 29 (8.5 g; 72\% yield from Example $2 \mathrm{a}$ compound). ${ }^{1} \mathrm{H} \mathrm{NMR}\left(\mathrm{CDCl}_{3}, \mathrm{~N}-\mathrm{H}\right.$ proton not located) $\delta$ $7.95(\mathrm{~d}, 2 \mathrm{H}, \mathrm{J}=8.8 \mathrm{~Hz}), 7.42(\mathrm{~d}, 2 \mathrm{H}, \mathrm{J}=8.8 \mathrm{~Hz}), 7.03(\mathrm{dd}, 1 \mathrm{H}, \mathrm{J}=5.7,2.8 \mathrm{~Hz}), 6.97(\mathrm{t}, 1 \mathrm{H}, \mathrm{J}=$ $9.4 \mathrm{~Hz}), 6.84-6.87(\mathrm{~m}, 1 \mathrm{H}), 4.94(\mathrm{~s}, 2 \mathrm{H}), 3.84(\mathrm{~s}, 2 \mathrm{H}), 3.72(\mathrm{~s}, 3 \mathrm{H}), 3.43(\mathrm{~s}, 2 \mathrm{H}), 2.43(\mathrm{~s}, 3 \mathrm{H})$ $\mathrm{ppm} ;{ }^{19} \mathrm{~F} \mathrm{NMR}\left(\mathrm{CDCl}_{3}\right) \delta-128.92 \mathrm{ppm} ;{ }^{13} \mathrm{C} \mathrm{NMR}\left(\mathrm{CDCl}_{3}\right) \delta 172.6,159.1,155.8(\mathrm{~d}, \mathrm{~J}=239.1$ $\mathrm{Hz}), 154.6,147.4,136.2$, 132.1, 129.01 (2C), 127.4 (2C), 127.1 (d, J = $15.3 \mathrm{~Hz}), 125.9,116.2$, $115.9(\mathrm{~d}, \mathrm{~J}=22.9 \mathrm{~Hz}), 114.8(\mathrm{~d}, \mathrm{~J}=7.6 \mathrm{~Hz}), 62.7,51.80,49.8,46.6,10.5$; HRMS for $\mathrm{C}_{21} \mathrm{H}_{21} \mathrm{ClFN}_{2} \mathrm{O}_{4}$, Calcd for $(\mathrm{M}+\mathrm{H})^{+}:$419.1174, Found: 419.1170.

\section{Methyl}

2-((5-((2-(4-chlorophenyl)-5-methyloxazol-4-yl)methoxy)

fluorobenzyl)(methoxycarbonyl)amino)acetate (26a). To a THF solution (15 mL) of Example 29 (3.9 g, $9.33 \mathrm{mmol}$ ) was added saturated aqueous $\mathrm{Na}_{2} \mathrm{CO}_{3}(10 \mathrm{~mL})$ and methyl chloroformate $(2.16 \mathrm{ml}, 28 \mathrm{mmol})$. The reaction was stirred at RT for $20 \mathrm{~min}$; at this time analytical HPLC indicated that the reaction was complete. The reaction was extracted with EtOAc $(3 \mathrm{x} 40 \mathrm{~mL})$. The combined organic extracts were dried $\left(\mathrm{MgSO}_{4}\right)$ and concentrated in vacuo. The residue was chromatographed $\left(\mathrm{SiO}_{2} ; 40 \mathrm{~g}\right.$ ISCO column; continuous gradient from 0 to $60 \% \mathrm{EtOAc}$ in hexanes) to compound afford 26a $(4.2 \mathrm{~g}, 95 \%)$ as an oil.

\section{$N$-(5-((2-(4-chlorophenyl)-5-methyloxazol-4-yl)methoxy)-2-fluorobenzyl)- $N$ -}

(methoxycarbonyl)glycine (3). To a solution of $26 \mathbf{a}(4.2 \mathrm{~g}, 8.8 \mathrm{mmol})$ in THF (40 mL) was added aqueous $\mathrm{NaOH}$ ( $46 \mathrm{~mL}$ of a $1 \mathrm{~N}$ solution; $46 \mathrm{mmol}$ ). The reaction was stirred for $2 \mathrm{~h}$ at RT, after which HPLC indicated that the reaction was complete. The reaction was acidified with aqueous $1 \mathrm{~N} \mathrm{HCl}$ to $\mathrm{pH} \sim 2-3$ and extracted with EtOAc $(3 \times 50 \mathrm{~mL})$. The combined organic extracts were dried $\left(\mathrm{MgSO}_{4}\right)$ and concentrated in vacuo to $-10 \mathrm{~mL}$ volume. $\mathrm{MeCN}(50 \mathrm{~mL})$ was added, and the mixture was concentrated at $40{ }^{\circ} \mathrm{C}$ to $-30 \mathrm{~mL}$ volume, then was stored at $0{ }^{\circ} \mathrm{C}$ overnight. The resulting white solid was filtered off, washed with $\mathrm{MeCN}(10 \mathrm{~mL})$ and dried in vacuo for $72 \mathrm{~h}$ to afford the title compound 3 (3.2 g). An additional $0.19 \mathrm{~g}$ of the title compound was obtained from the mother liquor by further recrystallization from $\mathrm{MeCN}$.

\section{$N$-(3-((2-(4-chlorophenyl)-5-methyloxazol-4-yl)methoxy)-5-fluorobenzyl)- $N$ - (methoxycarbonyl)glycine (4).}

${ }^{1} \mathrm{H}$ NMR (500 MHz, $\left.\mathrm{CDCl}_{3}\right) \delta 7.91(\mathrm{~d}, \mathrm{~J}=8.8 \mathrm{~Hz}, 2 \mathrm{H}), 7.41(\mathrm{~d}, \mathrm{~J}=8.8 \mathrm{~Hz}, 2 \mathrm{H}), 6.72-6.52(\mathrm{~m}$, $3 \mathrm{H}), 4.95(\mathrm{~s}, 2 \mathrm{H}), 4.52$ and $4.48(\mathrm{~s}, 2 \mathrm{H}$, rotamers), 3.98 and 3.90 (s, 2H, rotamers), 3.77 and 3.74 (s, 3H, methoxy group rotamers), 2.44 (s, 3H). Analytical HPLC: $100 \%$ pure, RT 4.70 min, ESHRMS calcd for $\mathrm{C}_{22} \mathrm{H}_{21} \mathrm{ClFN}_{2} \mathrm{O}_{6}(\mathrm{M}+\mathrm{H})^{+} 463.1072$, found 463.1080 . 
$N$-(3-((2-(4-chlorophenyl)-5-methyloxazol-4-yl)methoxy)-4-fluorobenzyl)- $N$ (methoxycarbonyl)glycine (5).

${ }^{1} \mathrm{H}$ NMR $\left(500 \mathrm{MHz}, \mathrm{CDCl}_{3}\right) \delta 7.91(\mathrm{~d}, \mathrm{~J}=8.8 \mathrm{~Hz}, 2 \mathrm{H}), 7.40(\mathrm{~d}, \mathrm{~J}=8.3 \mathrm{~Hz}, 2 \mathrm{H}), 7.17-6.77(\mathrm{~m}$, $3 \mathrm{H}$ ), 5.07 (s, 2H), 4.53 and 4.51 (s, $2 \mathrm{H}$, rotamers), 3.98 and 3.91 (s, 2H, rotamers), 3.80 and 3.75 (s, 3H, methoxy group rotamers), $2.46(\mathrm{~s}, 3 \mathrm{H})$. Analytical HPLC: > $98 \%$ pure, RT $5.35 \mathrm{~min}$, ES-HRMS calcd for $\mathrm{C}_{22} \mathrm{H}_{21} \mathrm{ClFN}_{2} \mathrm{O}_{6}(\mathrm{M}+\mathrm{H})^{+} 463.1072$, found 463.1089 .

(S)-N-(1-(3-((2-(4-chlorophenyl)-5-methyloxazol-4-yl)methoxy)phenyl)ethyl)- $N$ (methoxycarbonyl)glycine (6).

${ }^{1} \mathrm{H} \mathrm{NMR}\left(500 \mathrm{MHz}, \mathrm{CDCl}_{3}\right) \delta 7.92(\mathrm{~d}, \mathrm{~J}=8.8 \mathrm{~Hz}, 2 \mathrm{H}), 7.75$ (br s, $\left.1 \mathrm{H}\right), 7.44(\mathrm{~d}, \mathrm{~J}=8.3 \mathrm{~Hz}, 2 \mathrm{H})$, 7.28-7.23 (m, 1H), 7.0-6.89 (m, 3H), 5.0 (s, 2H), 3.88-3.62 (m, 6H), $2.46(\mathrm{~s}, 3 \mathrm{H}), 1.50$ (br s, 3H). Analytical HPLC: > $99 \%$ pure, RT 5.74 min. ES-HRMS calcd for $\mathrm{C}_{23} \mathrm{H}_{24} \mathrm{ClN}_{2} \mathrm{O}_{6}(\mathrm{M}+\mathrm{H})^{+}$ 459.1323, found 459.1344 .

\section{$N$-(2-fluoro-5-((5-methyl-2-(p-tolyl)oxazol-4-yl)methoxy)benzyl)- $N$ - (methoxycarbonyl)glycine (7).}

${ }^{1} \mathrm{H}$ NMR $\left(500 \mathrm{MHz}, \mathrm{CDCl}_{3}\right) \delta 7.86(\mathrm{~d}, \mathrm{~J}=7.7 \mathrm{~Hz}, 2 \mathrm{H}), 7.25(\mathrm{~d}, \mathrm{~J}=8.2 \mathrm{~Hz}, 2 \mathrm{H}), 7.04-6.82(\mathrm{~m}$, $3 \mathrm{H}), 4.96(\mathrm{~s}, 2 \mathrm{H}), 4.57$ and 4.52 (s, 2H, rotamers), 4.03 and 3.95 (s, 2H, rotamers), 3.75 and 3.71 (s, 3H, methoxy group rotamers), 2.43 (s, 3H), 2.39 (s, 3H). Analytical HPLC: $99 \%$ pure, RT 4.28 min, ES-HRMS calcd for $\mathrm{C}_{23} \mathrm{H}_{24} \mathrm{FN}_{2} \mathrm{O}_{6}(\mathrm{M}+\mathrm{H})^{+} 443.1618$, found 443.1626 .

\section{$N$-(2-fluoro-5-((2-(4-fluorophenyl)-5-methyloxazol-4-yl)methoxy)benzyl)- $N$ - (methoxycarbonyl)glycine (8).}

${ }^{1} \mathrm{H}$ NMR $\left(500 \mathrm{MHz}, \mathrm{CDCl}_{3}\right) \delta$ 7.94-7.91 (m, 2H), $7.06(\mathrm{t}, \mathrm{J}=8.3 \mathrm{~Hz}, 2 \mathrm{H})$, 7.05-6.76 (m, 3H), $4.87(\mathrm{~s}, 2 \mathrm{H}), 4.51$ and 4.46 (s, 2H, rotamers), 3.97 and 3.89 (s, 2H, rotamers), 3.70 and 3.66 (s, $3 \mathrm{H}$, methoxy group rotamers), 2.37 (s, 3H). Analytical HPLC: $100 \%$ pure, RT $3.60 \mathrm{~min}$. Anal. Calcd For $\mathrm{C}_{22} \mathrm{H}_{20} \mathrm{~F}_{2} \mathrm{~N}_{2} \mathrm{O}_{6}$ : C, 59.19; H, 4.51; F, 8.51; N, 6.27. Found: C, 58.95; H, 4.32; F, 8.50; N, 6.14 .

\section{$N$-(2-fluoro-5-((5-methyl-2-phenyloxazol-4-yl)methoxy)benzyl)- $N$ - (methoxycarbonyl)glycine (9).}

${ }^{1} \mathrm{H}$ NMR (500 MHz, $\mathrm{CDCl}_{3}$ ) $\delta 7.98(\mathrm{~d}, \mathrm{~J}=3.3 \mathrm{~Hz}, 2 \mathrm{H}), 7.43$ (br s, 3H), 7.05-6.82 (m, 3H), 4.95 (s, $2 \mathrm{H}), 4.57$ and 4.52 (s, $2 \mathrm{H}$, rotamers), 4.03 and 3.95 (s, $2 \mathrm{H}$, rotamers), 3.75 and 3.72 (s, $3 \mathrm{H}$, methoxy group rotamers), 2.43 (s, 3H). Analytical HPLC: $100 \%$ pure, RT 3.60 min, ES-HRMS calcd for $\mathrm{C}_{22} \mathrm{H}_{22} \mathrm{FN}_{2} \mathrm{O}_{6}(\mathrm{M}+\mathrm{H})^{+} 429.1462$, found 429.1450 . 
$N$-(2-fluoro-5-((5-methyl-2-(4-(trifluoromethyl)phenyl)oxazol-4-yl)methoxy)benzyl)- $N$ (methoxycarbonyl)glycine (10).

${ }^{1} \mathrm{H}$ NMR $\left(500 \mathrm{MHz}, \mathrm{CDCl}_{3}\right) \delta 8.11(\mathrm{~d}, \mathrm{~J}=8.3 \mathrm{~Hz}, 2 \mathrm{H}), 7.70(\mathrm{~d}, \mathrm{~J}=8.2 \mathrm{~Hz}, 2 \mathrm{H}), 7.04-6.85(\mathrm{~m}$, $3 \mathrm{H}), 4.96(\mathrm{~s}, 2 \mathrm{H}), 4.59$ and 4.55 (s, 2H, rotamers), 4.04 and 3.97 (s, 2H, rotamers), 3.77 and 3.73 (s, 3H, methoxy group rotamers), 2.47 (s, 3H). Analytical HPLC: $99 \%$ pure, RT 4.60 min, ESHRMS calcd for $\mathrm{C}_{23} \mathrm{H}_{21} \mathrm{~F}_{4} \mathrm{~N}_{2} \mathrm{O}_{6}(\mathrm{M}+\mathrm{H})^{+}$497.1336, found 497.1344.

\section{$N$-(2-fluoro-5-((5-methyl-2-(4-(trifluoromethoxy)phenyl)oxazol-4-yl)methoxy)benzyl)- $N$ - (methoxycarbonyl)glycine (11).}

1H NMR (500 MHz, $\left.\mathrm{CDCl}_{3}\right) \delta 8.02(\mathrm{~d}, \mathrm{~J}=8.3 \mathrm{~Hz}, 2 \mathrm{H}), 7.27(\mathrm{~d}, \mathrm{~J}=8.3 \mathrm{~Hz}, 2 \mathrm{H}), 7.04-6.82(\mathrm{~m}$, $3 \mathrm{H}), 4.94(\mathrm{~s}, 2 \mathrm{H}), 4.57$ and 4.53 (s, $2 \mathrm{H}$, rotamers), 4.03 and 3.96 (s, $2 \mathrm{H}$, rotamers), 3.75 and 3.71 (s, 3H, methoxy group rotamers), 2.44 (s, 3H). Analytical HPLC: $100 \%$ pure, RT $5.76 \mathrm{~min}$, ESHRMS calcd for $\mathrm{C}_{23} \mathrm{H}_{21} \mathrm{~F}_{4} \mathrm{~N}_{2} \mathrm{O}_{7}(\mathrm{M}+\mathrm{H})^{+}$513.1240, found 513.1243.

\section{$N$-(5-((2-(4-(tert-butyl)phenyl)-5-methyloxazol-4-yl)methoxy)-2-fluorobenzyl)- $N$ -} (methoxycarbonyl)glycine (12).

${ }^{1} \mathrm{H}$ NMR $\left(500 \mathrm{MHz}, \mathrm{CDCl}_{3}\right) \delta 7.91(\mathrm{~d}, \mathrm{~J}=8.2 \mathrm{~Hz}, 2 \mathrm{H}), 7.46(\mathrm{~d}, \mathrm{~J}=8.8 \mathrm{~Hz}, 2 \mathrm{H}), 7.06-6.82(\mathrm{~m}$, $3 \mathrm{H}$ ), 4.96 (s, 2H), 4.57 and 4.52 (s, 2H, rotamers), 4.04 and 3.95 (s, 2H, rotamers), 3.76 and 3.72 (s, 3H, methoxy group rotamers), $2.44(\mathrm{~s}, 3 \mathrm{H})$. Analytical HPLC: $100 \%$ pure, RT $5.49 \mathrm{~min}$, ESHRMS calcd for $\mathrm{C}_{26} \mathrm{H}_{30} \mathrm{FN}_{2} \mathrm{O}_{6}(\mathrm{M}+\mathrm{H})^{+} 485.2043$, found 485.2032 .

\section{$N$-(2-fluoro-5-((5-methyl-2-(4-(methylsulfonyl)phenyl)oxazol-4-yl)methoxy)benzyl)- $N$ - (methoxycarbonyl)glycine (13).}

${ }^{1} \mathrm{H}$ NMR $\left(500 \mathrm{MHz}, \mathrm{CDCl}_{3}\right) \delta 8.20(\mathrm{~d}, \mathrm{~J}=7.7 \mathrm{~Hz}, 2 \mathrm{H}), 8.02(\mathrm{~d}, \mathrm{~J}=8.2 \mathrm{~Hz}, 2 \mathrm{H}), 7.08-6.82(\mathrm{~m}$, $3 \mathrm{H}$ ), 4.96 (s, 2H), 4.60 and 4.56 (s, 2H, rotamers), 4.05 and 3.99 (s, 2H, rotamers), 3.78 and 3.74 (s, 3H, methoxy group rotamers), 3.09 (s, 3H), 2.48 (s, 3H). Analytical HPLC: $99 \%$ pure, RT 2.75 min, ES-HRMS calcd for $\mathrm{C}_{23} \mathrm{H}_{24} \mathrm{FN}_{2} \mathrm{O}_{8} \mathrm{~S}(\mathrm{M}+\mathrm{H})^{+} 507.1193$, found 507.1210.

\section{$N$-(5-((2-(4-chlorophenyl)-5-methyloxazol-4-yl)methoxy)-2-fluorobenzyl)- $N$ - (ethoxycarbonyl)glycine (14).}

${ }^{1} \mathrm{H}$ NMR $\left(500 \mathrm{MHz}, \mathrm{CDCl}_{3}\right) \delta 7.91(\mathrm{~d}, \mathrm{~J}=8.8 \mathrm{~Hz}, 2 \mathrm{H}), 7.41(\mathrm{~d}, \mathrm{~J}=8.8 \mathrm{~Hz}, 2 \mathrm{H}), 7.03-6.84(\mathrm{~m}$, $3 \mathrm{H}), 4.93(\mathrm{~s}, 2 \mathrm{H}), 4.57$ and $4.54(\mathrm{~s}, 2 \mathrm{H}$, rotamers), $4.25-3.96(\mathrm{~m}, 4 \mathrm{H}), 2.43(\mathrm{~s}, 3 \mathrm{H}), 1.27-1.23$ (m, 3H). Analytical HPLC: $100 \%$ pure, RT 4.89 min, ES-HRMS(m/e) calcd for $\mathrm{C}_{23} \mathrm{H}_{23} \mathrm{ClFN}_{2} \mathrm{O}_{6}$ $(\mathrm{M}+\mathrm{H})^{+}$477.1229, found 477.1241. 
$N$-(5-((2-(4-chlorophenyl)-5-methyloxazol-4-yl)methoxy)-2-fluorobenzyl)- $N$ (propoxycarbonyl)glycine (15).

${ }^{1} \mathrm{H}$ NMR $\left(500 \mathrm{MHz}, \mathrm{CDCl}_{3}\right) \delta 7.91(\mathrm{~d}, \mathrm{~J}=8.8 \mathrm{~Hz}, 2 \mathrm{H}), 7.41(\mathrm{~d}, \mathrm{~J}=8.8 \mathrm{~Hz}, 2 \mathrm{H}), 7.03-6.85(\mathrm{~m}$, $3 \mathrm{H}), 4.93(\mathrm{~s}, 2 \mathrm{H}), 4.58$ and $4.55(\mathrm{~s}, 2 \mathrm{H}$, rotamers $), 4.11-3.96(\mathrm{~m}, 4 \mathrm{H}), 2.42(\mathrm{~s}, 3 \mathrm{H}), 1.65-1.63(\mathrm{~m}$, 2H), $0.90(\mathrm{t}, \mathrm{J}=7.4 \mathrm{~Hz}, 3 \mathrm{H})$. Analytical HPLC: > $99 \%$ pure, RT $6.25 \mathrm{~min}$, ES-HRMS calcd for $\mathrm{C}_{24} \mathrm{H}_{25} \mathrm{ClFN}_{2} \mathrm{O}_{6}(\mathrm{M}+\mathrm{H})^{+} 491.1385$, found 491.1379.

$N$-(5-((2-(4-chlorophenyl)-5-methyloxazol-4-yl)methoxy)-2-fluorobenzyl)- $N-((3-$ fluoropropoxy)carbonyl)glycine (16).

${ }^{1} \mathrm{H}$ NMR (500 MHz, $\left.\mathrm{CDCl}_{3}\right) \delta 7.94(\mathrm{~d}, \mathrm{~J}=8.3 \mathrm{~Hz}, 2 \mathrm{H}), 7.42(\mathrm{~d}, \mathrm{~J}=7.2 \mathrm{~Hz}, 2 \mathrm{H}), 6.95-6.81(\mathrm{~m}$, $3 \mathrm{H}), 4.98(\mathrm{~s}, 2 \mathrm{H}), 4.56-4.28(\mathrm{~m}, 6 \mathrm{H}), 4.0-3.89(\mathrm{~d}, \mathrm{~J}=55 \mathrm{~Hz}, 2 \mathrm{H}), 2.45(\mathrm{~s}, 3 \mathrm{H}), 2.06-2.01(\mathrm{~m}$, 2H). Analytical HPLC: $100 \%$ pure, RT $5.67 \mathrm{~min}$, ES-HRMS(m/e) calcd for $\mathrm{C}_{24} \mathrm{H}_{24} \mathrm{ClF}_{2} \mathrm{~N}_{2} \mathrm{O}_{6}$ $(\mathrm{M}+\mathrm{H})^{+}$509.1291, found 509.1301.

$N$-(butoxycarbonyl)- $N$-(5-((2-(4-chlorophenyl)-5-methyloxazol-4-yl)methoxy)-2fluorobenzyl)glycine (17).

${ }^{1} \mathrm{H}$ NMR $\left(500 \mathrm{MHz}, \mathrm{CDCl}_{3}\right) \delta 7.92(\mathrm{~d}, \mathrm{~J}=8.3 \mathrm{~Hz}, 2 \mathrm{H}), 7.42(\mathrm{~d}, \mathrm{~J}=8.3 \mathrm{~Hz}, 2 \mathrm{H}), 7.03-6.85(\mathrm{~m}$, $3 \mathrm{H}), 4.93(\mathrm{~s}, 2 \mathrm{H}), 4.58$ and $4.54(\mathrm{~s}, 2 \mathrm{H}$, rotamers $), 4.15-3.95(\mathrm{~m}, 4 \mathrm{H}), 2.43(\mathrm{~s}, 3 \mathrm{H}), 1.62-1.62(\mathrm{~m}$, 2H), 1.37-1.31 ( $\mathrm{m}, 2 \mathrm{H}), 0.89(\mathrm{t}, \mathrm{J}=7.5 \mathrm{~Hz}, 3 \mathrm{H})$. Analytical HPLC: $99 \%$ pure, RT $6.72 \mathrm{~min}$, ES-HRMS calcd for $\mathrm{C}_{25} \mathrm{H}_{27} \mathrm{ClFN}_{2} \mathrm{O}_{6}(\mathrm{M}+\mathrm{H})^{+}$505.1542, found 505.1549.

\section{$N$-(5-((2-(4-chlorophenyl)-5-methyloxazol-4-yl)methoxy)-2-fluorobenzyl)- $N$ - (isobutoxycarbonyl)glycine (18).}

${ }^{1} \mathrm{H}$ NMR (500 MHz, $\left.\mathrm{CDCl}_{3}\right) \delta 7.93(\mathrm{~d}, \mathrm{~J}=8.8 \mathrm{~Hz}, 2 \mathrm{H}), 7.44(\mathrm{~d}, \mathrm{~J}=8.3 \mathrm{~Hz}, 2 \mathrm{H})$, 7.0-6.86 (m, $3 \mathrm{H}), 4.95(\mathrm{~s}, 2 \mathrm{H}), 4.60$ and $4.58(\mathrm{~s}, 2 \mathrm{H}$, rotamers), 4.05-3.90 (m, 4H), $2.45(\mathrm{~s}, 3 \mathrm{H}), 1.98-1.88($ $\mathrm{m}, 1 \mathrm{H}), 0.90(\mathrm{~d}, \mathrm{~J}=6.6 \mathrm{~Hz}, 6 \mathrm{H})$. Analytical HPLC: $>98 \%$ pure, RT $6.61 \mathrm{~min}$, ES-HRMS calcd for $\mathrm{C}_{25} \mathrm{H}_{27} \mathrm{ClFN}_{2} \mathrm{O}_{6}(\mathrm{M}+\mathrm{H})^{+}$505.1542, found 505.1536.

\section{$N$-(5-((2-(4-chlorophenyl)-5-methyloxazol-4-yl)methoxy)-2-fluorobenzyl)- $N$-((p-} tolyloxy)carbonyl)glycine (19).

${ }^{1} \mathrm{H}$ NMR $\left(500 \mathrm{MHz}, \mathrm{CDCl}_{3}\right) \delta 7.91(\mathrm{~d}, \mathrm{~J}=7.5 \mathrm{~Hz}, 2 \mathrm{H}), 7.39(\mathrm{~d}, \mathrm{~J}=7.5 \mathrm{~Hz}, 2 \mathrm{H}), 7.10(\mathrm{~d}, \mathrm{~J}=8.4$ $\mathrm{Hz}, 2 \mathrm{H}), 7.09-6.82(\mathrm{~m}, 5 \mathrm{H}), 4.94(\mathrm{~s}, 2 \mathrm{H}), 4.72$ and 4.63 (s, 2H, rotamers), 4.14 and 4.11 (s, 2H, rotamers), $2.42(\mathrm{~s}, 3 \mathrm{H}), 2.30(\mathrm{~s}, 3 \mathrm{H})$. Analytical HPLC: $99 \%$ pure, RT $5.90 \mathrm{~min}$, ES-HRMS calcd for $\mathrm{C}_{28} \mathrm{H}_{25} \mathrm{ClFN}_{2} \mathrm{O}_{6}(\mathrm{M}+\mathrm{H})^{+}$539.1385, found 539.1393. 
$N$-(5-((2-(4-chlorophenyl)-5-methyloxazol-4-yl)methoxy)-2-fluorobenzyl)- $N$-((4methoxyphenoxy)carbonyl)glycine (20).

${ }^{1} \mathrm{H}$ NMR $\left(500 \mathrm{MHz}, \mathrm{CDCl}_{3}\right) \delta 7.90(\mathrm{~d}, \mathrm{~J}=6.6 \mathrm{~Hz}, 2 \mathrm{H}), 7.38(\mathrm{~d}, \mathrm{~J}=8.3 \mathrm{~Hz}, 2 \mathrm{H}), 7.20-6.80(\mathrm{~m}$, $7 \mathrm{H}), 4.93(\mathrm{~s}, 2 \mathrm{H}), 4.70$ and 4.62 (s, $2 \mathrm{H}$, rotamers), 4.13 and 4.10 (s, $2 \mathrm{H}$, rotamers), $3.75(\mathrm{~s}, 3 \mathrm{H})$, 2.42 (s, 3H). Analytical HPLC: $98 \%$ pure, RT 5.46 min, ES-HRMS calcd for $\mathrm{C}_{28} \mathrm{H}_{25} \mathrm{ClFN}_{2} \mathrm{O}_{7}$ $(\mathrm{M}+\mathrm{H})^{+}$555.1334, found 555.1353.

\section{In vivo assays:}

Human apoA1 transgenic mice lipid studies: Male 6-8 week old human apoA1 transgenic mice were purchased from Jackson Laboratories (Jackson Lab, Bar Harbor, ME). These mice express human apoA1 gene under the control of the natural human apoA1 promoter. Mice were kept under a 12 hour light/dark standard light cycle and fed standard rodent chow (Harlan Teklad Rodent Diet 2018, S-5 6 Madison, WI) throughout the study. The dosing vehicle was 2\% Tween 80 (polyoxyethylenesorbitan monooleate) and 0.5\% CMC (carboxymethylcellulose) in 97.5\% Gibco distilled water.

Mice were randomly assigned into different treatment groups and weighed and dosed by oral gavage ( $5 \mathrm{ml} / \mathrm{kg}$ body weight) once a day in the morning with vehicle alone or with compound and allowed free access to food and water. The study duration was 10 days. After dosing on day 10, mice were fasted for 4 hours, sacrificed by $\mathrm{CO}_{2}$ asphyxiation and blood samples were collected in serumseparating tubes via cardiac puncture for lipid measurements. Livers were dissected out, weighed, and quickly frozen in liquid nitrogen for future RNA analysis. Human apoA1 concentration in serum was measured using the Apolipoprotein A1 kit (Polymedco).

Hamsters Lipid Studies: Male Syrian Golden Hamsters were acclimated to 12 hour light/dark reverse light cycle for 7 days with high fat diet, then dosed daily by oral gavage for 21 days while on the same diet. At the end of the experiment, blood samples were drawn retro-orbitally after an 18 hour fast and 24 hours after the last dose for the determination of serum lipid levels. Livers were dissected out for mRNA analysis. FPLC analysis of the serum from the experimental animals was analysed on a Superose 6HR 10/30 column installed on an AKTA purifier ${ }^{\circledR}$ (Amersham, software UNICORN V4.00.16) FPLC system. The diet (\% by weight) was $11.5 \%$ S67 coconut oil, $11.5 \%$ corn oil, $0.5 \%$ cholesterol and 5\% fructose using Purina 5001 as the base diet. The dosing vehicle was $0.5 \% \mathrm{CMC}$ with $2 \%$ Tween 80 .

Statistical analysis: Mean values for body-weight and food-consumption, values obtained from clinical laboratory tests, and organ weights of treated groups were compared to those of the control group using a Dunnett's test. Statistical comparisons across dose groups were performed using Tukey all pair comparison. A p value of were considered as significant changes. Data are expressed as mean + SEM. 


\section{Pharmacokinetics protocols for mouse and rat}

Mouse: The pharmacokinetics of BMS-711939 were determined in Balb/C mice. BMS-711939 was dissolved in a vehicle as a solution at approximately $1 \mathrm{mg} / \mathrm{mL}$. Animals were dosed by oral gavage (PO), and intravenous bolus (IV) administration via a tail-vein injection of dosing solution; six animals were dosed by each route. The IV dose was $5 \mathrm{mg} / \mathrm{kg}$ and the PO dose was $10 \mathrm{mg} / \mathrm{kg}$. Blood samples were collected, by retro-orbital bleed, at $0.05,0.5,1,4,6$, and $8 \mathrm{~h}$ after IV administration, and at $0.25,0.5,1,4,6$, and $8 \mathrm{~h}$ after $\mathrm{PO}$ administration.

Rat: The pharmacokinetics of BMS-711939 were determined in male Sprague-Dawley rats. Animals were surgically prepared, with indwelling jugular vein cannulae, and with carotid artery cannulae in the case of animals receiving an intravascular dose. Blood samples were collected from the jugular vein and centrifuged to separate plasma. The IV dose was $4 \mathrm{mg} / \mathrm{kg}$ and the PO dose was $8 \mathrm{mg} / \mathrm{kg}$. Blood samples were collected at $0.05,0.5,1,4,6$, and $8 \mathrm{~h}$ after IV administration, and at $0.25,0.5,1,4,6$, and $8 \mathrm{~h}$ after $\mathrm{PO}$ administration. 